\title{
Application of complex analysis to second order equations of mixed type
}

\author{
by Guo Chun Wen (Beijing)
}

\begin{abstract}
This paper deals with an application of complex analysis to second order equations of mixed type. We mainly discuss the discontinuous Poincaré boundary value problem for a second order linear equation of mixed (elliptic-hyperbolic) type, i.e. the generalized Lavrent'ev-Bitsadze equation with weak conditions, using the methods of complex analysis. We first give a representation of solutions for the above boundary value problem, and then give solvability conditions via the Fredholm theorem for integral equations. In [1], [2], the Dirichlet problem (Tricomi problem) for the mixed equation of second order $u_{x x}+\operatorname{sgn} y u_{y y}=0$ was investigated. In [3], the Tricomi problem for the generalized Lavrent'ev-Bitsadze equation $u_{x x}+\operatorname{sgn} y u_{y y}+A u_{x}+B u_{y}+C u=0$, i.e. $u_{\xi \eta}+a u_{\xi}+b u_{\eta}+c u=0$ with the conditions: $a \geq 0, a_{\xi}+a b-c \geq 0, c \geq 0$ was discussed in the hyperbolic domain. In the present paper, we remove the above assumption of [3] and obtain a solvability result for the discontinuous Poincaré problem, which includes the corresponding results in [1]-[3] as special cases.
\end{abstract}

I. Formulation of the discontinuous Poincaré problem for mixed equations of second order. Let $D$ be a simply connected bounded domain $D$ in the complex plane $\mathbb{C}$ with boundary $\partial D=\Gamma \cup L$, where $\Gamma(\subset\{y>0\}) \in C_{\mu}^{2}(0<\mu<1)$ with end points $z^{1}=0, z^{2}=2$ and $L=L_{1} \cup L_{2}, L_{1}=\{x=-y, 0 \leq x \leq 1\}, L_{2}=\{x=y+2,1 \leq x \leq 2\}$, and define $D_{1}=D \cap\{y>0\}, D_{2}=D \cap\{y<0\}$ and $z^{0}=1-i$. Using a conformal mapping, we may assume that $\Gamma=\{|z-1|=1$, $y \geq 0\}$.

We consider the second order linear equation of mixed type

$$
u_{x x}+\operatorname{sgn} y u_{y y}=A u_{x}+B u_{y}+\varepsilon C u+E \quad \text { in } D,
$$

where $A, B, C, E$ are functions of $z(\in D)$ and $\varepsilon$ is a real parameter. Its complex form is the following equation of second order:

1991 Mathematics Subject Classification: Primary 35M10.

Key words and phrases: discontinuous Poincaré problem, equations of mixed type, complex analytic method. 


$$
\begin{aligned}
u_{z \bar{z}} & =\operatorname{Re}\left[A_{1}(z) u_{z}\right]+\varepsilon A_{2}(z) u+A_{3}(z), \quad z \in D_{1}, \\
u_{\bar{z} \overline{z^{*}}}=\operatorname{Re}\left[A_{1}(z) u_{z}\right]+\varepsilon A_{2}(z) u+A_{3}(z), & z \in \bar{D}_{2},
\end{aligned}
$$

where

$$
\begin{aligned}
& z=x+i y, u_{z}=\frac{1}{2}\left[u_{x}-i u_{y}\right], u_{\bar{z}}=\frac{1}{2}\left[u_{x}+i u_{y}\right], u_{z \bar{z}}=\frac{1}{4}\left[u_{x x}+u_{y y}\right], \\
& u_{z^{*}}=\frac{1}{2}\left[u_{x}+i u_{y}\right]=u_{\bar{z}}, \quad u_{\bar{z} \overline{z^{*}}}=\frac{1}{2}\left[\left(u_{\bar{z}}\right)_{x}-i\left(\overline{u_{\bar{z}}}\right)_{y}\right]=\frac{1}{4}\left[u_{x x}-u_{y y}\right], \\
& A_{1}=\left\{\begin{array}{ll}
(A+i B) / 2 & \text { in } D_{1}, \\
(A-i B) / 2 & \text { in } D_{2},
\end{array} \quad A_{2}=C / 4, \quad A_{3}=E / 4 \quad \text { in } D .\right.
\end{aligned}
$$

Suppose that the equation (1.2) satisfies the following conditions:

Condition C. $A_{j}(z)(j=1,2,3)$ are measurable in $z \in D_{1}$ and continuous on $\bar{D}_{2}$ and their $L_{p}$ and $\alpha$-Hölder norms satisfy

$$
\begin{aligned}
& L_{p}\left[A_{j}, \bar{D}_{1}\right] \leq k_{0}, \quad j=1,2, \quad L_{p}\left[A_{3}, \bar{D}_{1}\right] \leq k_{1}, \\
& C_{\alpha}\left[A_{j}, \bar{D}_{2}\right] \leq k_{0}, \quad j=1,2, \quad C_{\alpha}\left[A_{3}, \bar{D}_{2}\right] \leq k_{1},
\end{aligned}
$$

where $p(>2), \alpha(0<\alpha<1), k_{0}, k_{1}$ are nonnegative constants.

In order to introduce the discontinuous Poincaré boundary value problem for the equation (1.2), let functions $a(z), b(z)$ have discontinuities of the first kind at $m+2$ distinct points $z_{0}=2, z_{1}, \ldots, z_{m}, z_{m+1}=0 \in \Gamma$, where $Z=\left\{z_{0}, z_{1}, \ldots, z_{m+1}\right\}$ is arranged according to the positive direction of $\Gamma$ and $m$ is a positive integer, and let $c(z)=O\left(\left|z-z_{j}\right|^{-\beta_{j}}\right)$ in the neighborhood of $z_{j}(j=0,1, \ldots, m+1)$ on $\Gamma$, where $\beta_{j}(j=0,1, \ldots, m+1)$ are small positive numbers. Define $\lambda(z)=a(x)+i b(x)$ and suppose $|a(x)|+|b(x)| \neq 0$; there is no harm in assuming that $|\lambda(z)|=1$ for $z \in \Gamma^{*}=\Gamma \backslash Z$. Suppose that $\lambda(z), c(z)$ satisfy the conditions

$$
\lambda(z) \in C_{\alpha}\left(\Gamma_{j}\right), \quad\left|z-z_{j}\right|^{\beta_{j}} c(z) \in C_{\alpha}\left(\Gamma_{j}\right), \quad j=0,1, \ldots, m+1,
$$

where $\Gamma_{j}$ is the open arc from $z_{j}$ to $z_{j+1}$ on $\Gamma$, with $z_{m+2}=2$, and $\alpha$ $(0<\alpha<1)$ is a constant.

Problem P. Find a continuously differentiable solution $u(z)$ of (1.2) in $D^{*}=\bar{D} \backslash \widetilde{Z}\left(\widetilde{Z}=\{x \pm y=2, y \leq 0\} \cup\left\{z_{1}, \ldots, z_{m+1}\right\}\right.$ or $\widetilde{Z}=\left\{z_{0}, \ldots, z_{m}\right\} \cup$ $\{x \pm y=0, y \leq 0\})$, which is continuous in $\bar{D}$ and satisfies the boundary conditions

$$
\begin{aligned}
& \frac{1}{2} \frac{\partial u}{\partial \nu}+\varepsilon \sigma(z) u=\operatorname{Re}\left[\overline{\lambda(z)} u_{z}\right]+\varepsilon \sigma(z) u \\
& =r(z)+Y(z) h(z), \quad z \in \Gamma, \quad u(0)=c_{0}, \\
& \frac{1}{2} \frac{\partial u}{\partial \nu}=\operatorname{Re}\left[\overline{\lambda(z)} u_{\bar{z}}\right]=r(z), \quad z \in L_{1} \text { or } L_{2},\left.\quad \operatorname{Im}\left[\overline{\lambda(z)} u_{\bar{z}}\right]\right|_{z=z^{0}}=b_{0}
\end{aligned}
$$


where $\nu$ is a vector at every point on $\Gamma \cup L_{j}(j=1$ or 2$), c_{0}, b_{0}$ are real constants, $\lambda(z)=a(x)+i b(x)=\cos (\nu, x)-i \cos (\nu, y)$ for $z \in \Gamma, \lambda(z)=a(x)+$ $i b(x)=\cos (\nu, x)+i \cos (\nu, y)$ for $z \in L_{j}(j=1$ or 2$)$, and $\lambda(z), r(z), c_{0}, b_{0}$ satisfy the conditions

$$
\begin{aligned}
& C_{\alpha}[\lambda(z), \Gamma] \leq k_{0}, C_{\alpha}[\sigma(z), \Gamma] \leq k_{0}, C_{\alpha}[r(z), \Gamma] \leq k_{2},\left|c_{0}\right|,\left|b_{0}\right| \leq k_{2}, \\
& C_{\alpha}\left[\lambda(z), L_{j}\right] \leq k_{0}, C_{\alpha}\left[\sigma(z), L_{j}\right] \leq k_{0}, C_{\alpha}\left[r(z), L_{j}\right] \leq k_{2}, j=1 \text { or } 2, \\
& \max _{z \in L_{1}} \frac{1}{|a(x)-b(x)|} \leq k_{0} \quad \text { or } \quad \max _{z \in L_{2}} \frac{1}{|a(x)+b(x)|} \leq k_{0},
\end{aligned}
$$

where $\alpha(1 / 2<\alpha<1), k_{0}$ and $k_{2}$ are nonnegative constants. Moreover, the functions $Y(z), h(z)$ are as follows:

$$
\begin{aligned}
& Y(z)=\eta \prod_{j=0}^{m+1}\left|z-z_{j}\right|^{\gamma_{j}}\left|z-z_{*}\right|^{l}, \quad z \in \Gamma^{*}, \\
& h(z)= \begin{cases}0, z \in \Gamma, & \text { if } K \geq-1 / 2, \\
h_{j} \eta_{j}(z), z \in \Gamma^{j}, & \text { if } K<-1 / 2,\end{cases}
\end{aligned}
$$

where $\Gamma^{j}(j=0,1, \ldots, m)$ are arcs on $\Gamma^{*}=\Gamma \backslash Z$ and $\Gamma^{j} \cap \Gamma^{k}=\emptyset$, $j \neq k, h_{j} \in J\left(J=\emptyset\right.$ if $K \geq-1 / 2 ; J=\left\{1, \ldots, 2 K^{\prime}-1\right\}$ if $K<-1 / 2$; $\left.K^{\prime}=[|K|+1 / 2]\right)$ are unknown real constants to be determined; $h_{1}=0$, $l=1$ if $2 K$ is odd, $z_{*}(\notin Z) \in \Gamma^{*}$ is any fixed point, and $l=0$ if $2 K$ is even; $\Gamma^{j}\left(j=1, \ldots, 2 K^{\prime}-1\right)$ are non-degenerate, mutually disjoint arcs on $\Gamma$, and $\Gamma^{j} \cap Z=\emptyset$ for $j=1, \ldots, 2 K^{\prime}-1 ; \eta_{j}(z)$ is a positive continuous function on the interior of $\Gamma^{j}$ such that $\eta_{j}(z)=0$ on $\overline{\Gamma \backslash \Gamma^{j}}$ and

$$
C_{\alpha}\left[\eta_{j}(z), \Gamma\right] \leq k_{0}, \quad j=1, \ldots, 2 K^{\prime}-1 ;
$$

and $\eta=1$ or -1 on $\Gamma_{j}\left(0 \leq j \leq m+1, \Gamma_{m+1}=(0,2)\right)$ as in [4], [6].

The above discontinuous Poincaré boundary value problem for (1.2) is called Problem P. Problem P for (1.2) with $A_{3}(z)=0$ for $z \in \bar{D}, r(z)=0$ for $z \in \Gamma \cup L_{j}(j=1$ or 2$)$ and $c_{0}=b_{0}=0$ will be called Problem $\mathrm{P}_{0}$.

Denote by $\lambda\left(z_{j}-0\right)$ and $\lambda\left(z_{j}+0\right)$ the left and right limits of $\lambda(z)$ as $z \rightarrow z_{j}(j=0,1, \ldots, m+1)$ on $\Gamma \cup L_{0}$, and

$$
\begin{array}{cc}
e^{i \phi_{j}}=\frac{\lambda\left(z_{j}-0\right)}{\lambda\left(z_{j}+0\right)}, \quad \gamma_{j}=\frac{1}{\pi i} \ln \left(\frac{\lambda\left(z_{j}-0\right)}{\lambda\left(z_{j}+0\right)}\right)=\frac{\phi_{j}}{\pi}-K_{j}, \\
K_{j}=\left[\frac{\phi_{j}}{\pi}\right]+J_{j}, \quad J_{j}=0 \text { or } 1, \quad j=0,1, \ldots, m+1 ;
\end{array}
$$

here $z_{m+1}=0, z_{0}=2, \lambda(z)=e^{i \pi / 4}$ on $L_{0}=(0,2)$ and $\lambda\left(z_{0}-0\right)=\lambda\left(z_{m+1}+0\right)$ $=\exp (i \pi / 4)$, or $\lambda(z)=e^{-i \pi / 4}$ on $L_{0}$ and $\lambda\left(z_{0}-0\right)=\lambda\left(z_{m+1}+0\right)=$ $\exp (-i \pi / 4) ;$ and $0 \leq \gamma_{j}<1$ when $J_{j}=0$ and $-1<J_{j}<0$ when $J_{j}=1$, 
$0 \leq j \leq m+1$. The quantity

$$
K=\frac{1}{2}\left(K_{0}+K_{2}+\ldots+K_{m+1}\right)=\sum_{j=0}^{m+1}\left(\frac{\phi_{j}}{2 \pi}-\frac{\gamma_{j}}{2}\right)
$$

is called the index of Problem $\mathrm{P}$ and Problem $\mathrm{P}_{0}$. If $\lambda(z)$ is continuous on $\Gamma \cup L_{0}$, then $K=\Delta_{\Gamma \cup L_{0}} \Gamma \arg \lambda(z) /(2 \pi)$ is a unique integer. If $\lambda(z)$ is not continuous on $\Gamma \cup L_{0}$, we may choose $J_{j}=0$ or 1 , hence the index $K$ is not unique.

Let $\beta_{j}+\gamma_{j}<1$ for $j=0,1, \ldots, m+1$. We can require that the solution $u(z)$ satisfies the condition $u_{z}=O\left(\left|z-z_{j}\right|^{-\delta_{j}}\right)$ in the neighborhood of $z_{j}$ $(j=0,1, \ldots, m+1)$ on $D^{*}$, where

$$
\begin{aligned}
\tau_{j} & = \begin{cases}\beta_{j}+\tau & \text { for } \gamma_{j} \geq 0, \text { and } \gamma_{j}<0, \beta_{j}>\left|\gamma_{j}\right|, \\
\left|\gamma_{j}\right|+\tau & \text { for } \gamma_{j}<0, \beta_{j} \leq\left|\gamma_{j}\right|,\end{cases} \\
\delta_{j} & = \begin{cases}2 \tau_{j}, & j=0, m+1, \\
\tau_{j}, & j=1, \ldots, m,\end{cases}
\end{aligned}
$$

and $\tau, \delta(<\tau)$ are small positive numbers. To ensure that the solution $u(z)$ of Problem $\mathrm{P}$ is continuously differentiable in $D^{*}$, we need to choose $\gamma_{1}>0$ or $\gamma_{2}>0$ respectively.

II. The representation of solutions for the oblique derivative problem for (1.2). Now we give the representation theorems for solutions of the equation (1.2)

Theorem 2.1. Let the equation (1.2) satisfy Condition $\mathrm{C}$ in $D_{1}$ and $\varepsilon=0, A_{2}(z) \geq 0$ in $D_{1}$, and $u(z)$ be a continuous solution of Problem $\mathrm{P}$ for (1.2) in $D_{1}$. Then $u(z)$ can be expressed as

$$
\begin{aligned}
& u(z)=U(z) \Psi(z)+\psi(z) \quad \text { in } D_{1}, \\
& U(z)=2 \operatorname{Re} \int_{0}^{z} w(z) d z+c_{0}, \quad w(z)=\Phi(z) e^{\phi(z)} \quad \text { in } D_{1},
\end{aligned}
$$

where $\psi(z), \Psi(z)$ are solutions of the equation (1.2) in $\bar{D}_{1}$ and of

$$
u_{z \bar{z}}-\operatorname{Re}\left[A_{1} u_{z}\right]-A_{2} u=0 \quad \text { in } D_{1},
$$

respectively and satisfy the boundary conditions

$$
\psi(z)=0, \quad \Psi(z)=1 \quad \text { on } \Gamma \cup L_{0} .
$$

Furthermore, $\psi(z), \Psi(z)$ satisfy the estimates

$$
\begin{gathered}
C_{\beta}^{1}\left[\psi, \bar{D}_{1}\right] \leq M_{1}, \quad\|\psi\|_{W_{p_{0}}^{2}\left(D_{1}\right)} \leq M_{1}, \\
C_{\beta}^{1}\left[\Psi, \bar{D}_{1}\right] \leq M_{2}, \quad\|\Psi\|_{W_{p_{0}}^{2}\left(D_{1}\right)} \leq M_{2}, \quad \Psi(z) \geq M_{3}>0, \quad z \in \bar{D}_{1},
\end{gathered}
$$


where $\beta(0<\beta \leq \alpha), p_{0}\left(2<p_{0} \leq p\right), M_{j}=M_{j}\left(p_{0}, k, \alpha, D_{1}\right)(j=1,2,3)$ are nonnegative constants, and $k=\left(k_{0}, k_{1}, k_{2}\right)$. Moreover, $U(z)$ is a solution of the equation

$$
U_{z \bar{z}}-\operatorname{Re}\left[A U_{z}\right]=0, \quad A=-(\ln \Psi)_{\bar{z}}+A_{1} \quad \text { in } D_{1},
$$

with $\operatorname{Im}[\phi(z)]=0$ for $z \in L_{0}=(0,2)$ and

$$
C_{\beta}\left[\phi, \bar{D}_{1}\right]+L_{p}\left[\phi_{\bar{z}}, \bar{D}_{1}\right] \leq M_{4},
$$

where $\beta(0<\beta \leq \alpha)$ and $M_{4}=M_{4}\left(p, \alpha, k_{0}, D\right)$ are nonnegative constants. Furthermore, $\Phi(z)$ is analytic in $D_{1}$, and $w(z)$ satisfies the boundary conditions

$$
\begin{aligned}
& \operatorname{Re}[\overline{\lambda(z)} w(z)]=r(z)-\operatorname{Re}\left[\overline{\lambda(z)}\left(\psi_{z}+\Psi_{z} U(z)\right)\right] \quad \text { on } \Gamma, \\
& \operatorname{Re}[\overline{\lambda(x)} w(x)]=s(x)-\operatorname{Re}\left[\overline{\lambda(x)}\left(\psi_{z}(x)+\Psi_{z}(x) U(x)\right)\right] \quad \text { on } L_{0}, \\
& \lambda(x)=\left\{\begin{array}{l}
1+i \text { or } \\
1-i,
\end{array} \quad x \in L_{0}=(0,2), \quad C_{\beta}\left[s(x), L_{0}\right] \leq k_{3},\right.
\end{aligned}
$$

and the estimate

$$
C_{\delta}\left[u(z), \bar{D}_{1}\right]+C_{\delta}\left[w(z) X(z), \bar{D}_{1}\right] \leq M_{5}\left(k_{1}+k_{2}+k_{3}\right) ;
$$

here $k_{3}$ is a nonnegative constant, $s(x)$ is given in (2.19) below, $X(z)=$ $\prod_{j=0}^{m+1}\left|z-z_{j}\right|^{\delta_{j}}, \delta_{j}(j=0,1, \ldots, m+1)$ are as stated in $(1.13)$, and $M_{5}=$ $M_{5}\left(p, \delta, k_{0}, D_{1}\right)$ is a nonnegative constant.

Pr o of. According to the method of proof in Chapter 3 of [6], the equations (1.2) and (2.2) have solutions $\psi(z), \Psi(z)$ respectively which satisfy the boundary condition (2.3) and the estimates (2.4) and (2.5). Setting

$$
U(z)=[u(z)-\psi(z)] / \Psi(z),
$$

it can be derived that $U(z)$ is a solution of (2.6) and can be expressed by the second formula in $(2.1)$, where $\phi(z)$ satisfies the estimate $(2.7), \Phi(z)$ is an analytic function in $D_{1}$, and $u(z), w(z)=U_{z}$ satisfy the boundary conditions $(2.8),(2.9)$ and the estimate (2.10). If $s(x)$ in (2.9) is a known function, then by the result in Chapter 4 of [6], the boundary value problem (2.8), (2.9) has a unique solution $w(z)$ as stated in (2.1).

Theorem 2.2. Suppose that the equation (1.2) satisfies Condition $\mathrm{C}$ and $\varepsilon=1, A_{2} \geq 0$ in $D_{1}$. Then any solution of Problem $\mathrm{P}$ for (1.2) can be expressed as

$$
u(z)=2 \operatorname{Re} \int_{0}^{z} w(z) d z+c_{0}, \quad w(z)=w_{0}(z)+W(z),
$$


where $w_{0}(z)$ is a solution of the following Problem A:

$$
\left\{\begin{array}{l}
w_{\bar{z}} \\
\bar{w}_{\overline{z^{*}}}
\end{array}\right\}=0 \quad \text { in }\left\{\begin{array}{l}
D_{1} \\
\bar{D}_{2}
\end{array}\right\}
$$

with the boundary conditions $(1.6),(1.7)\left(\sigma(z)=0, w_{0}(z)=u_{0 z}\right)$, and $W(z)$ has the form

$$
\begin{aligned}
& W(z)=w(z)-w_{0}(z), \quad W(z)=\widetilde{\Phi}(z) e^{\widetilde{\phi}(z)}+\widetilde{\psi}(z), \\
& \widetilde{\phi}(z)=\widetilde{\phi}_{0}(z)+T g=\widetilde{\phi}_{0}(z)-\frac{1}{\pi} \iint_{D_{1}} \frac{g(\zeta)}{\zeta-z} d \sigma_{\zeta}, \widetilde{\psi}(z)=T f \quad \text { in } D_{1}, \\
& \overline{W(z)}=\Phi(z)+\Psi(z), \quad \Psi(z)=\int_{2}^{\nu} g_{1}(z) d \nu e_{1}+\int_{0}^{\mu} g_{2}(z) d \mu e_{2}, \quad z \in D_{2} ;
\end{aligned}
$$

here $e_{1}=(1+i) / 2, e_{2}=(1-i) / 2, \mu=x+y, \nu=x-y$, and

$$
\begin{aligned}
& g(z)= \begin{cases}A_{1} / 2+\bar{A}_{1} \bar{w} /(2 w), & w(z) \neq 0, \\
0, & w(z)=0,\end{cases} \\
& f(z)=\operatorname{Re}\left[A_{1} \widetilde{\phi}_{z}\right]+A_{2} u+A_{3} \quad \text { in } D_{1}, \\
& g_{1}(z)=A \xi+B \eta+C u+D, \quad g_{2}(z)=A \xi+B \eta+C u+D \quad \text { in } \bar{D}_{2}, \\
& A=\left(\operatorname{Re} A_{1}+\operatorname{Im} A_{1}\right) / 2, \\
& B=\left(\operatorname{Re} A_{1}-\operatorname{Im} A_{1}\right) / 2, \quad C=A_{2}, \quad D=A_{3},
\end{aligned}
$$

where $\xi=\operatorname{Re} w+\operatorname{Im} w, \eta=\operatorname{Re} w-\operatorname{Im} w ;$ moreover, $\widetilde{\phi}_{0}(z)$ is an analytic function in $D_{1}$ such that $\operatorname{Im}[\widetilde{\phi}(x)]=0$ on $L_{0}=(0,2)$, and $\widetilde{\Phi}(z), \Phi(z)$ are solutions of (2.13) in $D_{1}, D_{2}$ respectively satisfying the boundary conditions

$$
\begin{aligned}
& \operatorname{Re}\left[\overline{\lambda(z)} e^{\widetilde{\phi}(z)} \widetilde{\Phi}(z)\right]=r(z)-\sigma(z) u(z)-\operatorname{Re}[\overline{\lambda(z)} \widetilde{\psi}(z)], \quad z \in \Gamma, \\
& \operatorname{Re}\left[\overline{\lambda(x)}\left(\widetilde{\Phi}(x) e^{\widetilde{\phi}(x)}+\widetilde{\psi}(x)\right)\right]=s(x), \quad x \in L_{0}, \\
& \operatorname{Re}[\overline{\lambda(x)} \Phi(x)]=-\operatorname{Re}[\overline{\lambda(x)} \Psi(x)], \quad z \in L_{0}, \\
& \operatorname{Re}[\overline{\lambda(z)} \Phi(z)]=-\operatorname{Re}[\overline{\lambda(z)} \Psi(z)], \quad z \in L_{1} \text { or } L_{2}, \\
& \operatorname{Im}\left[\overline{\lambda\left(z^{0}\right)} \Phi\left(z^{0}\right)\right]=-\operatorname{Im}\left[\overline{\lambda\left(z^{0}\right)} \Psi\left(z^{0}\right)\right] .
\end{aligned}
$$

Moreover, the solution $w_{0}(z)$ of Problem A for (2.13) satisfies

$$
\begin{array}{r}
C_{\delta}\left[u_{0}(z), \bar{D}\right]+C_{\delta}\left[w_{0}(z) X(z), \bar{D}_{1}\right]+C\left[w_{0}^{ \pm}(z) \widetilde{X}^{ \pm}(z), \bar{D}_{2}\right] \\
\leq M_{6}\left(k_{1}+k_{2}\right)
\end{array}
$$

(see [4]), where $X(z), \delta$ are as stated in $(2.10), M_{6}=M_{6}\left(\delta, k_{0}, D\right)$ is a 
nonnegative constant,

$$
w_{0}^{ \pm}(z)=\operatorname{Re} w_{0}(z) \mp \operatorname{Im} w_{0}(z), \quad \widetilde{X}^{ \pm}(z)=\prod_{j=1}^{2}\left|x \pm y-t_{j}\right|^{\delta_{j}}
$$

and

$$
u_{0}(z)=2 \operatorname{Re} \int_{0}^{z} w_{0}(z) d z+c_{0} .
$$

Proof. Let $u(z)$ be a solution of Problem P for (1.2), and $w(z)=u_{z}$, $u(z)$ be substituted in place of $w, u$ in (2.15). Thus the functions $g(z), f(z)$, $g_{1}(z), g_{2}(z)$, and $\widetilde{\psi}(z), \widetilde{\phi}(z)$ in $\bar{D}_{1}$ and $\Psi(z)$ in $\bar{D}_{2}$ in $(2.14),(2.15)$ can be determined. Moreover, we can find the solution $\widetilde{\Phi}(z)$ in $D_{1}$ and $\Phi(z)$ in $\bar{D}_{2}$ of (2.13) with the boundary conditions (2.16), where

$$
s(x)=\left\{\begin{array}{l}
r(x / 2) /[a(x / 2)-b(x / 2)] \text { or } \\
r(x / 2+1) /[a(x / 2+1)+b(x / 2+1)],
\end{array} \quad x \in L_{0} .\right.
$$

Thus

$$
w(z)= \begin{cases}\widetilde{\Phi}(z)^{\widetilde{\phi}(z)}+\widetilde{\psi}(z) & \text { in } D_{1}, \\ w_{0}(z)+W(z)=w_{0}(z)+\overline{\Phi(z)}+\overline{\Psi(z)} & \text { in } D_{2},\end{cases}
$$

is the solution of Problem A for the complex equation

$$
\left\{\begin{array}{l}
w_{\bar{z}} \\
\bar{w}_{\bar{z}^{*}}
\end{array}\right\}=\operatorname{Re}\left[A_{1} w\right]+A_{2} u+A_{3} \quad \text { in }\left\{\begin{array}{c}
D_{1} \\
D_{2}
\end{array}\right\}
$$

which can be expressed by the last formula in (2.12), and $u(z)$ is a solution of Problem $\mathrm{P}$ for (1.2) given by the first formula in (2.12).

III. The solvability conditions for the discontinuous Poincaré problem for (1.2). Set $w=u_{z}$ and consider the equivalent boundary value problem (Problem Q) for the mixed complex equation

$$
\begin{aligned}
& w_{\bar{z}}-\operatorname{Re}\left[A_{1}(z) w\right]=\varepsilon A_{2}(z) u+A_{3}(z), \quad z \in D_{1}, \\
& \bar{w}_{\bar{z}^{*}}-\operatorname{Re}\left[A_{1}(z) w\right]=A_{3}(z), \quad z \in D_{2}, \\
& u(z)=2 \operatorname{Re} \int_{0}^{z} w(z) d z+c_{0},
\end{aligned}
$$

with the boundary conditions

$$
\begin{aligned}
& \operatorname{Re}[\overline{\lambda(z)} w]=r(z)-\varepsilon \sigma(z) u+Y(z) h(z), \quad z \in \Gamma, \\
& \operatorname{Re}\left[\overline{\lambda(z)} u_{\bar{z}}\right]=r(z), \quad z \in L_{j}(j=1 \text { or } 2),\left.\quad \operatorname{Im}\left[\overline{\lambda(z)} u_{\bar{z}}\right]\right|_{z=z^{0}}=b_{0},
\end{aligned}
$$

where $c_{0}, b_{0}$ are real constants. By the result of [4], we can find the general 
solution of the following Problem $\mathrm{Q}_{1}$ for the mixed complex equation:

$$
\begin{aligned}
w_{\bar{z}}-\operatorname{Re}\left[A_{1}(z) w\right] & =A_{3}(z), \quad z \in D_{1}, \\
\bar{w}_{z^{*}}-\operatorname{Re}\left[A_{1}(z) w\right] & =A_{3}(z), \quad z \in D_{2},
\end{aligned}
$$

with the boundary conditions

$$
\begin{aligned}
& \operatorname{Re}[\overline{\lambda(z)} w(z)]=r(z)+Y(z) h(z), \quad z \in \Gamma, \\
& \operatorname{Re}[\overline{\lambda(z)} w(z)]=r(z), \quad z \in L_{j}(j=1 \text { or } 2),\left.\quad \operatorname{Im}[\overline{\lambda(z)} w(z)]\right|_{z=z^{0}}=b_{0},
\end{aligned}
$$

which can be expressed as

$$
\widetilde{w}(z)=w_{0}(z)+\sum_{k=1}^{2 K+1} c_{k} w_{k(z)}
$$

where $w_{0}(z)$ is a special solution of Problem $\mathrm{Q}_{1}$ and $w_{k}(z)(k=1, \ldots, 2 K+1)$ is the complete system of linearly independent solutions for the homogeneous problem corresponding to Problem $\mathrm{Q}_{1}$. Moreover, denote by $H_{2} u$ the solution of the following Problem $\mathrm{Q}_{2}$ for the complex equation:

$$
\begin{aligned}
w_{\bar{z}}-\operatorname{Re}\left[A_{1}(z) w\right] & =A_{2}(z) u, \quad z \in D_{1}, \\
\bar{w}_{\overline{z^{*}}}-\operatorname{Re}\left[A_{1}(z) w\right] & =A_{2}(z) u, \quad z \in D_{2},
\end{aligned}
$$

with the boundary conditions

$$
\begin{aligned}
& \operatorname{Re}[\overline{\lambda(z)} w(z)]=-\sigma(z) u+Y(z) h(z), \quad z \in \Gamma, \\
& \operatorname{Re}[\overline{\lambda(z)} w(z)]=0, \quad z \in L_{j}(j=1 \text { or } 2),\left.\quad \operatorname{Im}[\overline{\lambda(z)} w(z)]\right|_{z=z^{0}}=0,
\end{aligned}
$$

and the point conditions

$$
\operatorname{Im}\left[\overline{\lambda\left(a_{j}\right)} w\left(a_{j}\right)\right]=0, \quad j \in J= \begin{cases}\{1, \ldots, 2 K+1\}, & K \geq 0, \\ \emptyset, & K<0,\end{cases}
$$

where $a_{j} \in \Gamma \backslash Z$ are distinct points. It is easy to see that $H_{2}$ is a bounded operator mapping a function $u(z) \in \widetilde{C}^{1}(\bar{D})$ (i.e. $C(u, \bar{D})+C\left(X(z) u_{z}, \bar{D}_{1}\right)+$ $\left.C\left(\widetilde{X}(z) u_{z}^{ \pm}, \bar{D}_{2}\right)<\infty\right)$ to $w(z) \in \widetilde{C}_{\delta}(\bar{D})$ (i.e. $C_{\delta}(u, \bar{D})+C_{\delta}\left(X(z) w(z), \bar{D}_{1}\right)+$ $\left.C_{\delta}\left(\widetilde{X}(z) w^{ \pm}(z), \bar{D}_{2}\right)<\infty\right)$; here $X(z), \widetilde{X}(z)$ are as stated in Theorem 2.2. Furthermore, set

$$
u(z)=H_{1} w+c_{0}=2 \operatorname{Re} \int_{0}^{z} w(z) d z+c_{0}
$$

where $c_{0}$ is an arbitrary real constant. It is clear that $H_{1}$ is a bounded operator mapping $w(z) \in \widetilde{C}_{\delta}(\bar{D})$ to $u(z) \in \widetilde{C}^{1}(\bar{D})$. By Theorem 2.2, the function $w(z)$ can be expressed as an integral. From (3.9) and $w(z)=$ $\widetilde{w}(z)+\varepsilon H_{2} u$, we can obtain a nonhomogeneous integral equation $(K \geq 0)$ :

$$
u-\varepsilon H_{1} H_{2} u=H_{1} w(z)+c_{0}+\sum_{k=1}^{2 K+1} c_{k} H_{1} w_{k}(z) .
$$


Since $H_{1} H_{2}$ is a completely continuous operator in $\widetilde{C}^{1}(\bar{D})$, we can use the Fredholm theorem for the integral equation (3.10). Let

$$
\varepsilon_{j}(j=1,2, \ldots), \quad 0<\left|\varepsilon_{1}\right| \leq\left|\varepsilon_{2}\right| \leq \ldots,
$$

be the discrete eigenvalues for the homogeneous integral equation

$$
u-\varepsilon H_{1} H_{2} u=0 .
$$

Note that Problem $\mathrm{Q}$ for the complex equation (1.2) with $\varepsilon=0$ is solvable, hence $\left|\varepsilon_{1}\right|>0$.

We first discuss the case of $K \geq 0$. If $\varepsilon \neq \varepsilon_{j}(j=1,2, \ldots)$, then the nonhomogeneous integral equation (3.10) has a solution $u(z)$ and the general solution of Problem Q involves $2 K+2$ arbitrary real constants. If $\varepsilon$ is an eigenvalue of rank $q$ as in (3.11), then applying the Fredholm theorem, we obtain solvability conditions for the nonhomogeneous integral equation (3.10), which are a system of $q$ algebraic equations for $2 K+2$ arbitrary real constants. Letting $s$ be the rank of the corresponding coefficient matrix and $s \leq \min (q, 2 K+2)$, we can determine $s$ equalities in the $q$ algebraic equations, hence Problem Q for (1.2) has $q-s$ solvability conditions. When these conditions hold, then the general solution of Problem Q involves $2 K+2+q-s$ arbitrary real constants.

The case of $K<0$ can be similarly discussed. Thus we can state the following theorem.

Theorem 3.1. Suppose that the linear mixed equation (1.2) satisfies Condition C. Suppose $\varepsilon \neq \varepsilon_{j}(j=1,2, \ldots)$, where $\varepsilon_{j}(j=1,2, \ldots)$ are the eigenvalues of the homogeneous integral equation (3.12).

(1) If $K \geq 0$, then Problem $\mathrm{P}$ for (1.2) is solvable, and the general solution involves $2 K+2$ arbitrary real constants.

(2) If $K<0$, then Problem $\mathrm{P}$ for (1.2) has $-2 K-1-s$ solvability conditions and $s \leq 1$.

Suppose now that $\varepsilon$ is an eigenvalue of the homogeneous integral equation (3.12) with rank $q$.

(3) If $K \geq 0$, then Problem $\mathrm{P}$ for (1.2) has $q-s$ solvability conditions and $s \leq q$.

(4) If $K<0$, then Problem $\mathrm{P}$ for (1.2) has $-2 K-1+q-s$ solvability conditions and $s \leq \min (-2 K-1+q, 1+q)$.

Note that the Dirichlet problem (Problem D) for (1.2) with the boundary conditions

$$
u(z)=\phi(z) \quad \text { on } \Gamma \cup L_{j}(j=1 \text { or } 2)
$$

is a special case of Problem $\mathrm{P}$ with index $K=-1 / 2$. In fact, set $w=u_{z}$ in $D$. Then Problem D for the mixed equation (1.2) is equivalent to Problem A for 
the mixed equation (3.1) with the boundary condition (3.2) and the relation

$$
u(z)=2 \operatorname{Re} \int_{0}^{z} w(z) d z+c_{0}, \quad z \in D,
$$

where

$$
\begin{aligned}
& \lambda(z)=a+i b=\left\{\begin{array}{l}
\overline{i(z-1)}, \theta=\arg (z-1) \text { on } \Gamma, \\
\frac{1-i}{\sqrt{2}} \text { on } L_{1}, \text { or } \frac{1+i}{\sqrt{2}} \text { on } L_{2},
\end{array}\right. \\
& r(z)= \begin{cases}\phi_{\theta} & \text { on } \Gamma, \\
\phi_{x} / \sqrt{2} & \text { on } L_{1} \text { or } \phi_{x} / \sqrt{2} \text { on } L_{2},\end{cases} \\
& b_{0}=\operatorname{Im}\left[\frac{1+i}{\sqrt{2}} u_{\bar{z}}\left(z^{0}\right)\right]=\left.\frac{\phi_{x}-\phi_{x}}{\sqrt{2}}\right|_{z=z^{0}}=0, \text { or } \\
& b_{0}=\operatorname{Im}\left[\frac{1-i}{\sqrt{2}} u_{\bar{z}}\left(z^{0}\right)\right]=0, \\
& c_{0}=\phi(0) ;
\end{aligned}
$$

here $a=1 / \sqrt{2} \neq b=-1 / \sqrt{2}$ on $L_{1}$ or $a=1 / \sqrt{2} \neq-b=-1 / \sqrt{2}$ on $L_{2}$.

The index $K=-1 / 2$ of Problem $\mathrm{D}$ on $\partial D_{1}$ can be derived as follows: According to (3.13), the boundary conditions of Problem D in $D_{1}$ are

$$
\begin{array}{ll}
\operatorname{Re}[i(z-1) w(z)]=r(z)=\phi_{\theta}^{\prime} & \text { on } \Gamma, \\
\operatorname{Re}\left[\frac{1-i}{\sqrt{2}} w(x)\right]=\frac{\phi^{\prime}(x / 2)}{\sqrt{2}}, & x \in[0,2], \text { or } \\
\operatorname{Re}\left[\frac{1+i}{\sqrt{2}} w(x)\right]=s(x)=\frac{\phi^{\prime}(x / 2+1)}{\sqrt{2}}, & x \in[0,2] .
\end{array}
$$

It is clear that the possible discontinuity points of $\lambda(z)$ on $\partial D_{1}$ are $t_{1}=2$, $t_{2}=0$, and

$$
\begin{aligned}
& \lambda\left(t_{1}+0\right)=\frac{3 \pi}{2}, \quad \lambda\left(t_{2}-0\right)=\frac{\pi}{2}, \\
& \lambda\left(t_{1}-0\right)=\lambda\left(t_{2}+0\right)=e^{\pi i / 4}, \quad \text { or } \quad \lambda\left(t_{1}-0\right)=\lambda\left(t_{2}+0\right)=e^{7 \pi i / 4}, \\
& \frac{\lambda\left(t_{1}-0\right)}{\lambda\left(t_{1}+0\right)}=e^{-5 \pi i / 4}=e^{i \phi_{1}}, \quad \frac{\lambda\left(t_{2}-0\right)}{\lambda\left(t_{2}+0\right)}=e^{\pi i / 4}=e^{i \phi_{2}} \text { or } \\
& \frac{\lambda\left(t_{1}-0\right)}{\lambda\left(t_{1}+0\right)}=e^{\pi i / 4}=e^{i \phi_{1}}, \quad \frac{\lambda\left(t_{2}-0\right)}{\lambda\left(t_{2}+0\right)}=e^{-5 \pi i / 4}=e^{i \phi_{2}} .
\end{aligned}
$$

In order to insure the uniqueness of solutions of Problem D, we choose

$$
\begin{gathered}
-1<\gamma_{1}=\frac{\phi_{1}}{\pi}-K_{1}=-\frac{5}{4}-(-1)=-\frac{1}{4}<0,0 \leq \gamma_{2}=\frac{\phi_{2}}{\pi}-K_{2}=\frac{1}{4}<1, \text { or } \\
0 \leq \gamma_{1}=\frac{\phi_{1}}{\pi}-K_{1}=\frac{1}{4}<1,-1<\gamma_{2}=\frac{\phi_{2}}{\pi}-K_{2}=-\frac{5}{4}-(-1)=-\frac{1}{4}<0,
\end{gathered}
$$


thus

$$
\begin{aligned}
& K_{1}=-1, \quad K_{2}=0, \quad K=\left(K_{1}+K_{2}\right) / 2=-1 / 2, \quad \text { or } \\
& K_{1}=0, \quad K_{2}=-1, \quad K=\left(K_{1}+K_{2}\right) / 2=-1 / 2 .
\end{aligned}
$$

The unique solution $w(z)$ is continuous in $\bar{D}_{1} \backslash\{0,2\}$. For the second case, $w(z)$ is bounded in the neighbourhood of $t_{1}=2$, and the integral of $w(z)$ is bounded in the neighbourhood of $t_{2}=0$; for the first case, the integral of $w(z)$ is bounded in the neighbourhood of $t_{1}=2$, and $w(z)$ is bounded in the neighbourhood of $t_{2}=0$. If we require that the solution $w(z)$ is bounded in $\bar{D}_{1} \backslash\{0,2\}$, then it suffices to choose the index $K=-1$; in this case there is one solvability condition.

\section{References}

[1] A. V. Bitsadze, Differential Equations of Mixed Type, MacMillan, New York, 1964.

[2] - Some Classes of Partial Differential Equations, Gordon and Breach, New York, 1988.

[3] S. P. Pul'kin, The Tricomi problem for the generalized Lavrent'ev-Bitsadze equation, Dokl. Akad. Nauk SSSR 118 (1958), 38-41 (in Russian).

[4] G. C. Wen, Conformal Mappings and Boundary Value Problems, Amer. Math. Soc., Providence, R.I., 1992, 137-188.

[5] -, Oblique derivative problems for linear mixed equations of second order, Sci. in China Ser. A 41 (1998), 346-356.

[6] G. C. Wen and H. Begehr, Boundary Value Problems for Elliptic Equations and Systems, Longman, Harlow, 1990, 217-272.

Department of Mathematics

Peking University

Beijing 100871, China

E-mail: wengc@pku.edu.cn 